\title{
Cultura colaborativa y cultura participativa en la narrativa transmedia
}

Vicente Gosciola*

DOI: https://doi.org/10.17230/9789587206289ch3

Tatiane Eulália Mendes de Carvalho**

Jaqueline de Oliveira***

\section{Introducción}

El estudio sobre los diversos modos de colaboración en los proyectos transmedia pone de manifiesto la necesidad de establecer una diferencia entre la cultura colaborativa y la cultura participativa. Para ello es preciso identificar las iniciativas de colaboración que recurren o se encuentran en la narrativa transmedia, así como indagar en las formas en que la sociabilidad se organiza en movimientos colaborativos en la historia reciente de la comunicación. En ese orden de ideas, en este trabajo buscamos responder la pregunta de cuál es el alcance y el impacto de la cultura colaborativa en el contexto de la narrativa transmedia. Con esto en mente, partimos de la noción de "Zona Autónoma Temporal" de Hakim Bey para luego retomar los conceptos de Henry Jenkins y Jane McGonigal, entre otros autores que se han ocupado de la materia en cuestión.

El concepto de Zona Autónoma Temporal (TAZ, por sus siglas en inglés) de Hakim Bey (2011) no constituye un pensamiento político, crítico o filosófico a seguir. Es un pensamiento reflexivo, en respuesta a un Estado dominante en medio de la denominada globalización. Hakim Bey (seudónimo del historiador estadounidense Peter Lamborn Wilson) deja

* Licenciado en Educación Artística de la Facultad Paulista de Artes, con Especialización en Acción Educativa en Comunicación Social, magíster en Ciencias de la Comunicación de la Universidad de São Paulo y doctor en Comunicación y Semiótica de la Pontificia Universidad Católica de São Paulo. Profesor del Programa de Postgrado en Comunicación de la Universidad Anhembi Morumbi, São Paulo, Brasil. Correo electrónico: vicente.gosciola@gmail.com

** Licenciada en Comunicación Social-Periodismo de la Facultad Integrada Teresa de Ávila, magíster en Comunicación Social de la Universidad Metodista de São Paulo. Estudiante del Doctorado en Comunicación de la Universidad Anhembi Morumbi, São Paulo. Correo electrónico: tatieulalia@yahoo.com.br

*** Licenciada en Comunicación Social-Periodismo de la Facultad Paulus de Tecnología y Comunicación, con Especialización en Producción Audiovisual del Centro Universitario Senac, São Paulo. Estudiante de Maestría en Comunicación de la Universidad Anhembi Morumbi, São Paulo. Correo electrónico: jornalista.jack@gmail.com 
claro que no se trata de una rebelión u oposición directa a un Estado que detenta el control de la información como un todo, sino de una resistencia que piensa y actúa de modo autónomo y ocupa espacios aún no llenados por él. Se trata de una sociedad con la capacidad y los mecanismos para proponer sus propios cambios a partir de un objetivo común:

La TAZ es una especie de rebelión que no confronta al Estado directamente, una acción de guerrilla que libera un área (de tierra, de tiempo, de imaginación) y que se disuelve para rehacerse después, en otro lugar y otro momento, antes de que el Estado pueda aplastarla (Bey, 2011, p. 17). ${ }^{1}$

El internet y la expansión de las nuevas tecnologías en la web -definida por Bey como una estructura abierta, alternada y horizontal de intercambio de información en oposición a la $R e d$, que sería jerarquizada y elitista (la totalidad de todas las transferencias de información y de datos)contribuyó de manera relevante al desarrollo de herramientas de comunicación independiente para distintos movimientos sociales que antes no habían tenido voz. Con lo cual pudieron alcanzar un mayor número de audiencias sin que el Estado tuviera o pudiera obtener algún control sobre esas acciones:

La web no solo proporciona un apoyo logístico a la TAZ, también ayuda a crearla. En términos generales, podríamos decir que la TAZ "existe" tanto en el espacio de la información como en el "mundo real". La web puede compactar el tiempo, en forma de datos, en un "espacio" infinitesimal. Decimos que la TAZ, por ser temporal, no ofrece algunas de las ventajas de una libertad con una duración y localización más o menos estable. Pero la web ofrece una especie de sustituto en sí misma -pues ella puede informar a la TAZ, desde su inicio, con vastas cantidades de tiempo y espacio compactados en forma de datos que estaban siendo "infrautilizados" (Bey, 2011, p. 33).

De acuerdo con Bey, la TAZ ocupa tanto el mundo real como el virtual. Está presente en ambos espacios y los movimientos sociales pueden y deben seguir la misma lógica de ocupación haciéndose cada vez más presentes en un universo que pertenece a todos por igual, y donde el flujo de datos e informaciones pueden ser pirateados dentro y fuera de la web. La interacción y la conexión entre las redes de los diversos movimientos

${ }^{1}$ Todas las traducciones de los textos citados en portugués e inglés son nuestras. 
sociales y organismos independientes se ha organizado y ganado cada vez más espacio por medio del activismo digital lo que se traduce en importantes acciones fuera del espacio virtual al conectar el ciberespacio con el espacio urbano. Los vehículos de comunicación masiva dejaron de ser la única fuente de información para dar espacio a nuevos vehículos. En palabras de Castells (2013):

La comunicación masiva se basa en redes horizontales de comunicación interactiva que generalmente son difíciles de controlar por parte de los gobiernos o las empresas. Además, la comunicación digital es multimodal y permite la referencia constante a un hipertexto global de informaciones cuyos componentes pueden ser remezclados por el actor que comunica de acuerdo con proyectos de comunicación específicos. La autocomunicación masiva proporciona la plataforma tecnológica para la construcción de la autonomía del actor social, ya sea individual o colectivo, en relación con las instituciones de la sociedad (p. 12).

En tiempos de conectividad lo colectivo gana fuerza, y en el nuevo modo de generar contenido -resultante de la convergencia digital-surge la llamada cultura participativa y la cultura de la colaboración. Sin embargo, hay diferencias entre la cultura participativa y la cultura colaborativa en lo que se refiere a los procesos de expansión o construcción de contenido, como lo señalan en sus obras Henry Jenkins y Jane McGonigal.

\section{Cultura participativa y cultura colaborativa}

Con el advenimiento de la web 2.0, la presencia de las redes sociales y el auge de los smartphones, hubo un cambio en el proceso de comunicación y en el hábito de consumir información, en el que el ciudadano deja de ser pasivo (recibe y procesa la información) y pasa a ser un agente de producción de contenido. Jenkins (2009) le da a este momento el nombre de cultura de convergencia.

El investigador estadounidense presenta tres conceptos para explicar la convergencia: inteligencia colectiva, cultura participativa y convergencia de los medios de comunicación. En 1978, Roxanne Hiltz y Murray Turoff fueron los primeros en acuñar el término "inteligencia colectiva" para describir un sistema computacional (Malone \& Bernstein, 2015, p. 6). En 1993, el texto de Hiltz y Turoff fue retomado por Howard Rheingold para referirse a las primeras experiencias de socialización y comunicación 
en Arpanet, la predecesora de la Internet (Rheingold, 2000, p. 113). La evolución de la aplicación del término se da con Derrick de Kerckhove en 1995, cuando recupera la idea de inteligencia colectiva de un sistema computacional colectivo y conectado y lo expande a la sociedad en una nueva ecología de las redes, constatando ciertas formas de agrupación colectiva que parecían sincronizadas "como si cualquier persona supiera lo que otras personas están haciendo" (Kerckhove, 1997, p. 252). Es en esta lógica que tenemos, la convergencia de los medios de comunicación como un flujo de información entre diversas plataformas. Representa, así, una modificación cultural que incentiva al usuario a buscar nuevas informaciones y a efectuar conexiones con el contenido expuesto en otros medios.

El origen de la cocreación, sea en forma de cultura participativa o cultura colaborativa, ciertamente se dio con las vanguardias artísticas. Así, por ejemplo, en una ponencia sobre el acto creativo presentada en la Convención de la American Federation of Arts, en abril de 1957, Marcel Duchamp exponía la siguiente reflexión:

Después de todo, el acto creativo no es realizado solo por el artista; el espectador pone a la obra en contacto con el mundo exterior, descifrando e interpretando su calificación interna y, así, añade su contribución al acto creativo. Esto es aún más obvio cuando la posteridad da un veredicto final y a veces restituye a los artistas olvidados (Duchamp, 1957, citado en Lebel, 1959, p. 78).

Un pionero del arte colaborativo fue László Moholy-Nagy, en una experiencia que refleja nuevos comportamientos y nuevas técnicas, derivados sobre todo de la revolución en las comunicaciones:

En 1922, pedí por teléfono a una fábrica de lacas cinco pinturas para porcelana esmaltada. Tenía la tabla de colores de la fábrica, con la que bosquejaba las pinturas sobre un papel cuadriculado. Al otro lado del teléfono, el supervisor de la fábrica tenía el mismo tipo de papel, dividido en cuadros. Él trazó las formas dictadas en la posición correcta. (Fue como jugar ajedrez por correspondencia). Una de las pinturas me la enviaron en tres formatos diferentes, para que pudiera analizar las diferencias en las relaciones de color causadas por la ampliación y la reducción (Moholy-Nagy, 1947, p. 79).

Con todo, las intervenciones artísticas colectivas en tecnologías de comunicación y computación tuvieron lugar a partir de la década de los 
setenta, como el trabajo de Kit Galloway y Sherrie Rabinowitz en el Satellite Arts Project'77 "A space with no geographical boundaries" (1977), llevando al extremo, para la época, el concepto de la imagen como lugar, gracias al uso del satélite para la transmisión y recepción en vivo entre dos puntos distantes en Estados Unidos, donde los artistas creaban colectivamente y se veían en una performance "telecolaborativa". ${ }^{2}$ Aunque aquí el trabajo artístico sea menos estudiado que los flujos sociales de contenido entre las redes digitales, Nicolas Bourriaud apunta a la relevancia de aquel experimento:

El surgimiento de nuevas tecnologías, como el Internet y el multimedia, indica un deseo colectivo de crear nuevos espacios de convivencia y de inaugurar nuevos tipos de contacto con el objeto cultural; así, a la 'sociedad del espectáculo' le seguiría entonces la sociedad de los figurantes, en la que cada uno encontraría en los canales de comunicación [...] la ilusión de una democracia interactiva (Bourriaud, 2009, p. 36).

Jenkins (2009) cree que el factor cultural es el que conduce todo el proceso de la convergencia, y destaca el concepto de cultura participativa. Nosotros consideramos que es necesario explicar más detalladamente el concepto. Por tanto, buscamos las diferencias entre las definiciones de cultura participativa y de cultura colaborativa en el contexto social y mediático.

Como punto de partida, tomamos las acepciones de colaboración y participación del Diccionario Aurelio (Ferreira, 2008) para entender el sentido de las palabras y sus acciones. La colaboración hace referencia a "desarrollar un trabajo en común, con una o más personas en una obra y contribuir con el desarrollo del trabajo, efectuar un trabajo de cooperación" (2008, p. 244). La participación, en el mismo diccionario (2008, p. 611), se refiere a "hacer saber [de algo], comunicar, informar, compartir, tomar parte, asociarse por el sentimiento o pensamiento -del dolor, la alegría, el luto, etc.-".

Observamos y asumimos, entonces, que existe una diferencia de acciones entre las acepciones citadas. Colaborar es construir en conjunto y participar es estar presente o interactuar de alguna forma, no necesariamente contribuir.

Jenkins, Ford y Green (2014) afirman que los debates sobre la cultura participativa surgieron antes que las nuevas plataformas de comunicación.

\footnotetext{
${ }^{2}$ http://www.ecafe.com/getty/SA/
} 
A la mitad del siglo XIX, las personas escribían a mano o mecanografiaban contenidos sobre temas de política, cultura o de asuntos cotidianos que luego entregaban a la población en forma impresa, haciendo circular así la información. Es algo similar a lo que hoy se etiqueta en las "redes sociales" (Jenkins, Purushotma, Weigel, Clinton, \& Robison, 2009) por el hecho de distribuir contenidos y al mismo tiempo compartirlos para quienes deseen tener acceso a ellos.

Jenkins, Purushotma, Weigel, et al. (2009) destacan que la historia de la cultura participativa tuvo avances en las décadas del 60 y $70 \mathrm{con} \mathrm{el}$ movimiento de contracultura, el crecimiento de los medios (radio y TV) y la relación entre la política, la cultura y los ciudadanos en la democracia. Hubo una expansión de las producciones culturales de diversas comunidades en línea, como por ejemplo el podcasting que llevó la radio a la internet y le dio participación a otros grupos diferentes para producir y hacer circular contenidos.

Al describir el concepto de cultura participativa, en su libro Cultura de la convergencia, Henry Jenkins (2009, p. 29) dice que la convergencia de los medios de comunicación en múltiples plataformas va más allá de la tecnología, pues para que esto suceda se necesita una transformación cultural en la mente de los consumidores. Es decir, el consumidor construye la propia historia y la fragmenta de acuerdo con su cotidianidad:

La expresión cultura participativa contrasta con nociones más antiguas sobre la pasividad de los espectadores de los medios de comunicación. En lugar de hablar sobre productores y consumidores de medios como roles separados, podemos ahora considerarlos como participantes que interactúan de acuerdo con un nuevo conjunto de reglas, que ninguno de nosotros entiende por completo (Jenkins, 2009, p. 30).

Jenkins destaca que cada consumidor recibe la información de forma individual y participa de acuerdo con su cultura emergente. Por eso, un contenido mediático puede ser compartido de acuerdo con la cultura, la comunidad y el modo de participación del individuo, independientemente de su posición geográfica, gracias al cambio del proceso de comunicación que pasa de la distribución a la circulación. Este modelo incrementa la participación de la cultura del público, que ya no es visto como consumidor sino como agente capaz de moldear, compartir y remezclar el contenido mediático. 
Clay Shirky (2011), por su parte, plantea que en el siglo xx la comunicación giraba en torno al consumo y la producción, lo cual cambió cuando las personas empezaron a producir y compartir lo que consumían. Este investigador describe que la revolución se origina con la máquina digital, el smartphone, por ejemplo, con el que se puede compartir, producir y consumir contenido, acciones básicas en el producto digital. Shirky resalta que la cultura participativa le dio voz a quien no podía opinar; ganar seguidores a un cantante desconocido; publicar sus textos (en forma de blogs o en las redes sociales digitales) a escritores que no contaban con el apoyo de una editorial; obtener recursos por medio de financiación abierta a las ONG, etc.; es decir, los individuos anónimos se convirtieron en productores de contenidos (aficionados, pero productores, al fin y al cabo).

Otro punto importante para este cambio es el escenario económico contemporáneo, en el cual el consumo se destaca y proporciona productos con más facilidad para la sociedad. En el libro Confronting the Challenges of Participatory Culture, Jenkins et al. (2009) exponen el resultado de una investigación realizada con niños, jóvenes y adultos en Estados Unidos, sobre la cultura participativa en las escuelas y las redes sociales, y presentan cinco características que definen dicho concepto dentro del grupo estudiado (2009, p. xi):

1 Pocos obstáculos para la expresión artística y el compromiso cívico.

2 Gran soporte para la creación de contenidos y recursos compartidos.

3 Distintos tipos de tutorías proporcionadas por personas que cuentan con determinada experticia.

4 Productores que creen en la importancia de sus contribuciones.

5 Personas que sienten una conexión social con otras (o al menos, se preocupan por lo que otras personas piensan sobre lo que crearon).

En este contexto, Jenkins et al. (2009) explican que no todos deben contribuir con la creación de contenido, pero todos saben que pueden aportar cuando lo deseen. Otro dato señalado por la investigación es que la cultura participativa se subdivide en cuatro categorías -afiliaciones, expresiones, soluciones colaborativas y circulaciones-, descritas de la siguiente forma:

Afiliaciones: Las asociaciones, formales e informales, en comunidades en línea centradas en varias formas de medios, tales como Friendster, Facebook, MySpace, foros, metagaming y clanes de jugadores. 
Expresiones: Producir nuevas formas creativas como muestreo digital, desmonte y modificación, videos de fans, fan fiction, sines y mash-ups.

Solución colaborativa de problemas: Trabajar en conjunto por equipos -formales e informales- para completar tareas y desarrollar nuevos conocimientos; por ejemplo, a través de Wikipedia y juegos de realidad alternativa. Circulaciones: Darle forma al flujo de medios, como podcasting o blogging (Jenkins et al., 2009, pp. xi-xii).

La investigación también aborda la brecha de participación entre adolescentes, jóvenes y adultos, puesto que estos tienen cierta dificultad en producir su contenido mientras que entre los niños y jóvenes tal acción es común en sus rutinas. Jenkins et al. (2009) señalan que es necesario incluir el desarrollo de habilidades de la cultura participativa en la educación con el fin de lograr una interacción entre el conocimiento, la producción de conocimiento y la enseñanza para el nuevo escenario de la comunicación. Ellos clasifican dichas habilidades como se cita a continuación:

Juego: La capacidad de experimentar con el entorno como forma de resolución de problemas.

Desempeño: La habilidad para adoptar identidades alternativas con el propósito de improvisar y descubrir.

Simulación: La capacidad de interpretar y construir modelos dinámicos de procesos del mundo real.

Apropiación: La capacidad de tomar y remezclar contenido multimedia de manera significativa.

Multitarea: La capacidad de explorar y analizar el entorno y desplazar el foco a los detalles más sobresalientes.

Cognición distribuida: La capacidad de interactuar de manera significativa con herramientas que amplían las capacidades mentales.

Inteligencia colectiva: La capacidad de unir conocimiento y comparar observaciones con otras personas hacia un objetivo común.

Juicio: La capacidad de evaluar la confiabilidad y credibilidad de diferentes fuentes de información.

Navegación transmedia: La capacidad de seguir el flujo de historias e informaciones en múltiples modalidades.

Redes: La capacidad de buscar, sintetizar y diseminar información.

Negociación: La capacidad de transitar en diversas comunidades, discernir y respetar múltiples perspectivas y comprender y seguir modelos alternativos (Jenkins et al., 2009, p. xiv). 
En el libro Cultura de la conexión, escrito por Jenkins, Ford y Green (2014, p. 198), se plantea que la cultura participativa evoluciona, así como los ciudadanos, de tal suerte que empiezan a surgir nuevos comportamientos y acciones en la Red debido al marketing, las ciencias políticas, los estudios culturales, la educación y la antropología digitales. En tal sentido los autores contrastan los siguientes aspectos: observación frente a participación periférica; resistencia y participación; audiencia y público; participación y colaboración; escuchar y oír.

Jenkins et al. (2014) contrastan la observación frente a la participación periférica debido al hecho de que muchos consumidores interactúan observando lo que sucede en la Red sin crear su contenido. Este es un público adulto y su forma de contribuir consiste en compartir y disfrutar de los contenidos a su disposición. Son resistentes a la industria mediática convencional, que tiene miedo de las acciones participativas de los ciudadanos. Para explicar esta realidad, Jenkins et al. usan los términos fans y fandoms. Los fans son individuos aislados, que comparten y crean grupos de discusión sobre un determinado tema de su interés, y los fandoms son grupos de personas o aficionados a un determinado asunto, un colectivo.

En esa línea, destacamos la participación frente a la colaboración, aspecto importante para el objetivo de nuestra investigación. De acuerdo con Jenkins et al. (2014) la colaboración es una relación compleja, crea nuevas oportunidades y herramientas, rompe las reglas y está más cerca del sector corporativo debido a su forma de acción:

La participación en una comunidad de marca o de fans puede o no ser un modo de influir en la cultura y puede o no ser un modo de intensificar el compromiso del público con el producto o marca. Los colaboradores son cómplices de los regímenes dominantes de poder, aunque a menudo también usen su incorporación en ese sistema para redirigir las energías o reecaminar los recursos de éstos (p. 220).

Jenkins, et al. (2014) plantean que la cultura participativa, en cambio, no solo está involucrada con la comunidad, aun con el desafío que representa el crecimiento social y la cultura, sino que también le proporciona más fuerza a los compromisos activistas.

Estos investigadores indican que el ciudadano colaborador es connivente con los grandes medios, pero muchas veces se vale de esta situación para infiltrarse en el medio, tener acceso y poder colaborar con la creación 
del contenido. En cuanto a la diferencia entre oír y escuchar, el primer aspecto está ligado a las estrategias de marketing en tanto que el segundo evidencia la razón por la cual el público quiere interactuar con una empresa y su contenido. Por lo tanto, las empresas necesitan escuchar, ya que oír es solo recibir un mensaje, mientras que escuchar es conversar con su público.

En contraste, Jane McGonigal (2012) afirma que la colaboración es una forma especial de trabajo conjunto que no significa únicamente unir fuerzas o alcanzar un objetivo, sino construir colectivamente algo que sería difícil crear por una sola persona (p. 268). A su vez, la colaboración requiere tres tipos de esfuerzo concentrado: cooperación (actuar en pro de un objetivo común), coordinación (armonizar y coordinar aptitudes) y cocreación (crear colectivamente nuevos productos). La cocreación es considerada por McGonigal el principal factor para la colaboración:

Casi todo puede ser creado a través de la colaboración: una experiencia de grupo, un recurso de conocimiento, una obra de arte. Cada vez más, los jugadores colaboran para crear todos estos resultados. De hecho, colaboran incluso cuando compiten entre sí para ganar. De manera creciente, los jugadores colaboran incluso cuando están jugando solos (McGonigal, 2012, p. 269).

Michael Tomasello (citado por McGonigal, 2012, p. 271) dice que la acción de compartir es definida por la habilidad de participar, junto a otras personas, de una actividad colaborativa con unos objetivos comunes. Y afirma que la práctica de seguir metas inusuales y trabajar en equipo está ligada al instinto natural de todo ser humano.

McGonigal lleva a cabo su investigación en el contexto de los videojuegos, y encuentra como evidencia el desarrollo creciente de plataformas digitales para juegos basados en un sistema de creación colaborativa en el que los jugadores necesitan crear contenido propio para ayudar a los demás jugadores. No obstante, aclara que:

Las habilidades colaborativas están en ascenso en todo el mundo, incluso entre los no jugadores. Desde la diseminación de la alfabetización básica en la Internet y los smartphones hasta la Web 2.0, en rápida expansión, y el know how del crowdsourcing, las personas, en todas partes, están cada vez más conectadas, mejorando de manera significativa sus habilidades de cooperar, coordinar y crear en grupo. En este sentido, los jugadores 
son solo la expresión de una tendencia social y tecnológica más amplia, hacia una colaboración mayor (McGonigal, 2012, p. 277).

Como lo muestra esta autora, dicho sistema de colaboración hace crecer la industria, genera nuevos productos y conocimientos y fortalece la cooperación, la coordinación y la cocreación. A su vez, McGonigal acuña el concepto de "superpoderes colaborativos", que consiste en una nueva habilidad con la cual se modifica el entendimiento de lo que es humanamente posible y que va más allá del modelo tradicional de enseñanza. Se trata de acciones sociables en ambientes on-line que buscan organizar ambientes caóticos, alejarse de viejas metas para crear otras nuevas, así como ampliar nuevas comunidades colaborativas (no solo en el juego) mediante la utilización de la inteligencia colectiva y la producción colaborativa.

Las consideraciones de Jane McGonigal sobre la cultura colaborativa están en sintonía con la definición del concepto de colaboración del Diccionario Aurelio (Ferreira, 2008), y presenta divergencias con la noción de cultura participativa de Jenkins, incluso cuando éste habla de la colaboración y describe los pasos para involucrar a la sociedad en la convergencia cultural.

\section{La transmedia y la cultura colaborativa}

La transmedia consiste en el desarrollo de una narrativa de forma simultánea en diferentes plataformas, cada una de las cuales ofrece un contenido exclusivo de la historia principal. Se trata del concepto de transmedia storytelling, una nueva estética que surge como respuesta a la convergencia de los medios de comunicación y sus recursos de interacción y que genera estrategias de propagación de contenido por medio de la cultura participativa. Según Jenkins:

La transmedia implica el procesamiento de nuevos tipos de historias y argumentos que emergen dentro de una cultura de convergencia, expresando ideas de maneras que explotan las oportunidades y los recursos representados por el nuevo paisaje de los medios. En otras palabras, implica la capacidad de leer y escribir en todos los modos de expresión disponibles (2009, p. 89).

Este modo de contar y propagar historias en múltiples plataformas que propicia la narrativa transmedia aumenta la capacidad de participación y 
colaboración del espectador y consumidor de diversos contenidos. Hay un universo de contenidos susceptible de ser creado para cada género de producción, como por ejemplo la TV, el cine, los videojuegos y las obras seriadas de ficción y no ficción. La construcción de este universo se propaga gracias a la participación activa de las comunidades de los fans de dichas producciones.

En las plataformas digitales, como las redes sociales virtuales, es posible involucrar a diferentes comunidades denominadas fanfictions y fandoms en otros espacios de conexión y convergencia de contenido, como blogs, chats y páginas especializadas. Por lo tanto, el flujo de producción de la narrativa transmedia como un todo no sigue una lógica predefinida o determinada por el productor del contenido creado. La expansión de la narrativa de modo colaborativo se da por la audiencia y puede alcanzar impactos de grandes proporciones, lo que trae como resultado la longevidad de determinado universo.

Para Suzanne Scott (2013), la idea de una cultura colaborativa cobra relevancia en los procesos de autoría colaborativa de los proyectos de narrativa transmedia, a los que alude en el siguiente comentario de su ensayo sobre el papel del autor de fanboy en la narrativa transmedia:

A diferencia de lo que ocurre en una historia transmedia, la discusión de este ensayo sobre el autor de fanboy y su función debe considerarse solo como un punto de entrada a un tema mucho más amplio y rico que está en expansión y desarrollo. Primero, este ensayo no describe cómo el autor podría proceder de manera diferente en las franquicias transmedia creadas por compañías no comerciales, o cuando surgen en medios distintos a la televisión. Por ejemplo, una franquicia transmedia cultivada en torno a un cómic, un medio que se define en gran parte por la 'visión' de autoría unificada y colaborativa creada entre un escritor y un artista, ciertamente complicaría algunas de las afirmaciones de este ensayo. En segundo lugar, en ningún momento de este ensayo menciono las extensiones transmedia que requieren un alto grado de colaboración entre creadores y consumidores, como los juegos de realidad alterna (ARG), que solo pueden prosperar si los maestros/creadores y los jugadores/fans trabajan juntos (2013, pp. 50-51).

Ahora bien, si la cultura colaborativa ha existido siempre (en forma de comentarios y acciones entre amigos, o grupos de estudiantes), es con la internet que se ha expandido y ha tomado un cuerpo nunca antes 
dimensionado. Pero lo que los sitios de crowdsourcing y crowdfunding posibilitan, por ejemplo, es algo que rebasa todo lo que se ha hecho en términos de cultura colaborativa. $Y$ es una tendencia que debe aumentar, porque no siempre las instituciones dan cuenta de todas las demandas de la sociedad. No somos pequeñas aldeas interconectadas por estrechas carreteras; vivimos en un planeta con más de 7000 millones de personas que pueden comunicarse a través de la internet. Si la globalización convencional -basada en best sellers y majors productoras de entretenimiento- no puede dar cuenta de cada demanda particular, entonces les queda a los interesados en un asunto común reunirse y, vía web, materializar sus deseos.

Por ejemplo, el cine es una obra colectiva, pero está organizada por un concentrador de inversiones: el productor ejecutivo. Cuando un proyecto no tiene espacio en el campo de visión de los inversores, necesita esa red colaborativa, no solo para crear, producir, distribuir y exhibir, sino también para financiar. En ese sentido el proyecto Life in a Day ${ }^{3}$ de Ridley y Tony Scott fue realizado en 2011. Cualquier persona podía enviar por YouTube un video con imágenes de lo que ocurrió el 24 de julio de 2010 respondiendo a tres preguntas: "Lo que hay en tu bolsillo", “¿Qué amas?" y “¿De qué sientes miedo?”. Se enviaron 80000 videos de 192 países, de los cuales fueron seleccionados 33 cortos, editados en una película de 95 minutos.

También en 2011 surge Bar Karma,${ }^{4}$ una serie web de ciencia ficción colaborativa dirigida por Will Wright. Fue un experimento de entretenimiento en crowdsourced, que transformó historias escritas por fans en episodios de 30 minutos producidos con equipo y elenco profesionales. Los miembros publicaban sus loglines en la comunidad del Bar Karma. Las historias seleccionadas se expandían a storyboards. Las más votadas por los miembros entraron en la línea de producción para la temporada de doce episodios.

También está el caso de HitRECord on TV, ${ }^{5}$ una serie de televisión creada en 2014 por Joseph Gordon-Levitt. La serie desarrolla un tema único por episodio enfatizando los conceptos de celebridad web y de la

\footnotetext{
${ }^{3} \mathrm{http}: / /$ www.imdb.com/title/tt1687247/?ref_=nv_sr_1

${ }^{4} \mathrm{http}: / /$ www.imdb.com/title/tt1786211/?ref- $=\mathrm{fn}_{-} \mathrm{tt}-\mathrm{tt} 1$

${ }^{5} \mathrm{http}: / /$ www.imdb.com/title/tt3453566/?ref_=nv_sr_1
} 
historia del arte. Cada episodio fue producido a partir de contribuciones de miembros de la comunidad HitRECord en todo el mundo -cartas, performances en vivo, dibujos animados, etcétera-.

Es innegable que ese proceso cultural colectivo es refutado por los inversores, empresarios y consultores de la denominada industria cultural. Sin embargo, para la sociedad como un todo y para la evolución de la cultura es una iniciativa altamente beneficiosa. 
Actualmente, el alcance de las plataformas de medios y la interacción con la tecnología es cada vez más rápida y natural; a medida que el acceso a la información se amplía en las redes de todo el mundo, creamos cada vez más formas de propagar contenidos afines. El "compromiso digital" deja de tener un contexto social, para pasar a generar estrategias de negocio. Tanto la cultura de participación como la de colaboración están ligadas y son más visibles en la transmedialidad.

A partir de los aportes de Jenkins, se presupone que el término de cultura colaborativa está vinculado con la producción del contenido de una obra en conjunto con su realizador o poseedor de su propiedad intelectual, que tras su creación es propagada por la cultura participativa, la cual contribuye a compartirla en las distintas plataformas de medios en línea y fuera de línea. Por lo tanto, lo que se produce en conjunto con la audiencia es colaborativo y, después de compartido por la interacción de la audiencia, se convierte en participativo. Ambos procesos forman parte del flujo de producción transmediática y son de suma importancia para generar los desdoblamientos y el compromiso que se orienta a la narrativa transmedia como un todo; además, fomentan la transmisión de los contenidos generados de modo exclusivo para cada plataforma de medios, lo que posibilita un mayor alcance de acuerdo con las preferencias individuales de las personas.

En McGonigal, si bien su comprensión sobre la participación y la colaboración es relativamente cercana a la de Jenkins, las atribuciones de cada uno de los dos aspectos son más precisas y son expuestas de un modo más técnico, pues delimita las funciones de producción compartida y producción desarrollada en conjunto, la cocreación; por tanto, se trata de dos maneras diferentes de abordar el mismo asunto, con enfoques dirigidos a determinado tipo de público, pues McGonigal está familiarizada con el universo de los videojuegos y Jenkins con la sociedad en sí y sus formas de vivir la cultura de la convergencia y el compromiso de la audiencia en las producciones transmediáticas. 
En suma, a partir de lo expuesto hasta aquí, las definiciones de cultura participativa y de cultura colaborativa son específicas y diferentes una de la otra, y poseen unos roles socioculturales claros y distintos, aunque ambas están presentes de modo relevante e indispensable en cualquier producción que se identifique como narrativa transmedia. 


\section{Referencias}

Bey, H. (2011). TAZ: Zona autônoma temporária (3ª ed.). São Paulo, Brasil: Conrad.

Bourriaud, N. (2009). Estética relacional. São Paulo, Brasil: Martins.

Castells, M. (2013). Redes de indignação e esperança: movimentos sociais na era da internet. Rio de Janeiro, Brasil: Paz e Terra.

Ferreira, A. B. D. H. (2008). Novo dicionário Aurélio da língua portuguesa. Rio de Janeiro, Brasil: Nova fronteira.

Jenkins, H. (2009). Cultura da convergência (2 $2^{\mathrm{a}}$ ed.). São Paulo, Brasil: Aleph.

Jenkins, H., Purushotma, R., Weigel, M., Clinton, K., \& Robison, A. J. (2009). Confronting the Challenges of Participatory Culture: Media education for the 21st century. Cambridge, MA, Estados Unidos; Londres, Inglaterra: MIT Press. Disponible en https://bit.ly/2ENQPLi

Jenkins, H., Ford, S., \& Green, J. (2014). Cultura da conexão: criando valor e significado por meio da mídia propagável. São Paulo, Brasil: Aleph.

Kerckhove de, D. (1997). A Pele da Cultura: uma investigação sobre a nova realidade electrónica. Lisboa, Portugal: Relógio D’Água.

Lebel, R. (1959). Marcel Duchamp. Nueva York, NY: Paragraphic.

Malone, M., \& Bernstein, T. (Eds.) (2015). Handbook of Collective Intelligence. Cambridge, MA: MIT Press.

McGonigal, J. (2012). A realidade em jogo. Rio de Janeiro, Brasil: Best Seller.

Moholy-Nagy, L. (1947). The New Vision and Abstract of an Artist. Nueva York, NY: Wittenborn.

Rheingold, H. (2000). The Virtual Community: homesteading on the electronic frontier. Cambridge, MA: MIT Press.

Scott, S. (2013). Who's Steering the Motership? The Role of the Fanboy Auteur in Transmedia Storytelling. En A. Delwiche, \& J. Jacobs 
Henderson (Eds.), The Participatory Cultures Handbook (pp. 43-52). Nueva York, NY: Routledge.

Shirky, C. (2011). A cultura da participação: criatividade e generosidade no mundo conectado. Rio de Janeiro: Zahar. 